\title{
Administration of Casirivimab and Imdevimab for Patient Undergoing Hemodialysis with Coronavirus Disease 2019: A Case Report and Literature Review
}

Yoshinosuke Shimamura ( $\nabla$ yshimamura.tkh@gmail.com )

Teine Keijinkai Medical Center https://orcid.org/0000-0003-0278-6900

Takuto Maeda

Norihihito Moniwa

Hideki Takizawa

Research Article

Keywords: COVID-19, REGEN-COV, hemodialysis.

Posted Date: February 4th, 2022

DOI: https://doi.org/10.21203/rs.3.rs-1122914/v1

License: (9) (i) This work is licensed under a Creative Commons Attribution 4.0 International License.

Read Full License 


\section{Abstract}

The neutralizing monoclonal antibody combination of casirivimab and imdevimab (REGEN-COV) has been reported to reduce viral load, hospitalization, and death among patients with coronavirus disease 2019 (COVID-19). However, cases of patients undergoing hemodialysis who are treated with REGEN-COV are rare. A 37-year-old Japanese patient undergoing hemodialysis presented with fever and was diagnosed with COVID-19 pneumonia. The patient had end-stage kidney disease due to diabetic nephropathy and was administered a single dose of REGEN-COV. The patient's symptoms rapidly resolved and was discharged without complications. Our case demonstrates the effectiveness of REGENCOV as a treatment for patients with mild COVID-19 undergoing hemodialysis.

\section{Background}

In Japan, the administration of a combination of casirivimab and imdevimab (REGEN-COV) received approval from the Japanese Ministry of Health, Labour, and Welfare (MHLW)inJuly 2021. ${ }^{1}$ REGEN-COV is an antibody cocktail therapy that targets the spike protein of severe acute respiratory syndrome coronavirus 2 (SARS-CoV-2) and prevents virus entry into human cells. Patients with mild to moderate coronavirus disease 2019 (COVID-19) who have chronic kidney disease are clinically indicated for this treatment. ${ }^{2}$ Arandomized controlled trial demonstrated that the administration of REGEN-COV reduced viral load, COVID-19-related hospitalization, and death. ${ }^{3}$ Although there is a case reportingthe safety of REGEN-COV in kidney transplant recipients with COVID-19, ${ }^{4}$ reports of REGEN-COV administration for patients undergoing hemodialysisare limited. ${ }^{5}{ }^{6}$ Herein, we report a case of a Japanese patient undergoing hemodialysis with mild COVID-19 who was treated with REGEN-COV. This report highlights the importance of the early use of REGEN-COV in patients with COVID-19 undergoing hemodialysis to prevent disease progression and reduce the duration of clinical symptoms.

\section{Case Presentation}

A 37-year-old Japanese patientundergoinghemodialysis presented with fever, chills, sore throat, and headache. There was no complaint of arthralgia, myalgia,dysgeusia, or anosmia. The first dose of the COVID-19 vaccine(Pfizer-BioNTech) was administered to the patientthree days before the presentation;exposure to a family member infected with COVID-19 had occurred two days before the presentation. The patient's comorbidities included end-stage kidney disease, obesity (body mass index,31.2 kg/m²), diabetes mellitus, and hypertension.Upon examination, his respiratory rate was 24 breaths/min, heart rate 79 beats/min, blood pressure 149/95 mmHg, oxygen saturation $98 \%$, and body temperature $37.9{ }^{\circ} \mathrm{C}$; other findings included tachypnea. Laboratory findings included elevated white blood cell count $\left(9,470 / \mu \mathrm{l}\right.$ [normal: 3,300-8,600 / $\mu$ l]), normal platelet count $\left(16.3 \times 10^{9} / \mathrm{L}\right.$ [normal: 15.8 $\left.34.8 \times 10^{9} / \mathrm{l}\right]$ ), and D-dimer of $<0.5 \mu \mathrm{g} / \mathrm{ml}$ (normal range: $<1.0 \mu \mathrm{g} / \mathrm{ml}$ ). The results of the SARS-CoV-2 antigen test of salivary specimens (FUJIREBIO, Inc., Tokyo, Japan) was $48.6 \mathrm{pg} / \mathrm{ml}$ (normal range: $<0.67$ $\mathrm{pg} / \mathrm{ml}$ ). The patient had a positive result for the reverse transcriptase-polymerase chain reaction test for 
SARS-CoV-2, andplain computed tomography of the chest showed a focal ground-glass opacity in the right lower lobe (Figure). The patient had multiple comorbidities and was at risk of progression to severe COVID-19, thus,we recommended usingREGEN-COV. He consented to the recommended treatment. After admission, a single dose of REGEN-COV (casirivimab $1200 \mathrm{mg}$ and imdevimab $1200 \mathrm{mg}$ )was administered without any hypersensitivity reaction. On the second day, his respiratory rate normalized to 16 breaths/min, oxygen saturationto $98 \%$, andbody temperature to $36.5^{\circ} \mathrm{C}$. Other clinical symptoms, including chills, sore throat, and headaches, were also resolved. His symptoms rapidlyresolved, and he was discharged after 10 days.Afterdischarge, the patient did not experience any fatigue orweakness.

\section{Discussion}

In the present case, we made two notable observations. First, this case illustrates that earlyadministration of REGEN-COV is effective for patientsundergoing hemodialysis to prevent progression tosevere illness. Patients with end-stage kidney disease, particularly those undergoinghemodialysis, are known to have a high risk of infection and mortality from COVID-19. ${ }^{7}$ Previous randomized controlled trials ${ }^{3}$ demonstrated thatparticipants administered with REGEN-COV had reduced risk of COVID-19-related hospitalization and death; however, evidence on the efficacy of REGEN-COV amongpatients with end-stage kidney disease is still limited. ${ }^{4-6} \mathrm{~A}$ paper reported that kidney transplant recipients administered withREGEN-COV did not require mechanical respiratory support or escalation of care to the intensive care unit. ${ }^{4}$ Severalcases from Japanhave reportedthe effectiveness of REGEN-COV for patients undergoinghemodialysis. ${ }^{5,6}$ These cases are summarized in Table, one concerned a man in his 40swith end-stage kidney disease (ESKD) due to diabetic nephropathy, and another concerned a man in his 50s with ESKD due to autosomal dominant polycystic kidney disease, both were treated successfully with REGEN-COV. In this case, the patient showed rapid resolution after administration of REGEN-COV and did not require supplemental oxygen therapy.It is less likely that the possibility of the prevention of disease progression is due to the effectiveness of the first dose of the COVID-19 vaccinebecause the vaccine usually takes several weeks to elicit an optimal humoral response. ${ }^{8}$ Moreover, patients undergoing hemodialysis have a low seroconversion rate after administration of the COVID-19 vaccine. ${ }^{9}$ In a study of 69 patients undergoing hemodialysis who wereadministered the COVID-19 vaccine, an anti-spike protein antibody was detected in $33 \%$ of patients after the first dose and $86 \%$ after the second dose. ${ }^{9}$

Second, this case indicates that REGEN-COV shortened the duration of the patient's clinical symptoms. This is compatible with the results of a previous trial showing that participants who were administeredREGEN-COV showed rapid resolution and a shorter duration of hospitalization than those who were administered a placebo among symptomatic outpatients with COVID-19. ${ }^{3}$ In the United States, REGEN-COV was given emergency use authorization by the Food and Drug Administration and was available for non-hospitalized patients with COVID-19. ${ }^{2}$ In contrast,we administered REGEN-COV because the Japanese MHLWallowed it to be used only for hospitalized patients. ${ }^{1}$ On October 1, 2021, the ministry changed its policy to ensure that outpatients with COVID-19 could be administer REGEN-COV if they could contact a physician immediately to assist hospital admission if their condition worsened. ${ }^{10}$ 


\section{Conclusions}

We report a case of COVID-19 pneumonia in a Japanese patient undergoing hemodialysis who was treated with REGEN-COV. Our case illustrates the benefits of REGEN-COV at an early stage of infectionto prevent disease progression and reduce symptom duration. We suggest that clinicians should consider REGEN-COV as a treatment option when providing medical care for patients with COVID-19 undergoing hemodialysis.

\section{Abbreviations}

COVID-19: coronavirus disease 2019

ESKD: end-stage kidney disease

MHLW: the Japanese Ministry of Health, Labour, and Welfare

REGEN-COV: casirivimab and imdevimab

SARS-CoV-2: severe acute respiratory syndrome coronavirus 2

\section{Declarations}

Ethics Approval and Consent to Participants: This study was approved by the Internal Review Board of the Teine Keijinkai Medical Center (IRB Approval No. 2-020134-00)and was carried out in accordance with the Declaration of Helsinki. Informed consent for publication was individually obtained from all participants included in the study.

Consent for Publication: All co-authors approved this submission. The patients consented to publish their information details.

Availability of Data and Materials: The datasets used and/or analyzed during the current study are available from the corresponding author on reasonable request.

Competing Interests: The authors declare that they have no competing interests.

Funding: Not applicable.

Acknowledgments: We would like to thank Editage for English language editing.

\section{References}

1. The Japanese Ministry of Health, Labour, and Welfare. Distribution of the combination of casirivimab and indevimab for medical facilities in Japan. [updated July 20, 2021;cited November 05, 202]. Available from: https://www.mhlw.go.jp/content/000808472.pdf 
2. The U.S. Food and Drug Administration. Fact sheet for health care providers emergency use authorization (EUA) of REGEN-COV ${ }^{\mathrm{TM}}$ (casirivimab and imdevimab). [ cited November 05, 2021]. Available from: https://www.fda.gov/media/145611/download

3. Weinreich DM, Sivapalasingam S, Norton S, et al. REGEN-COV antibody combination and outcomes in outpatients with Covid-19. N Engl J Med. 2021 Sep 29;NEJMoa2108163. DOI: 10.1056/NEJMoa2108163.

4. Liu EC, Lee JH, Loo A, et al. Casirivimab-Imdevimab (REGN-COV2) for mild and moderate SARS-CoV2 infection in kidney transplant recipients. Kidney Int Rep. 2021 Sep 6; 6(11): 2900-2902. DOI: 10.1016/j.ekir.2021.08.032.

5. Ohtsubo S, Minami M, Ohshima $\mathrm{Y}$, et al. An experience of neutralizing monoclonal antibody treatment in a hemodialysis patient with SARS-CoV-2 infection. Nihontouseki-ikaizasshi (in Japanese). [updated August 20, 2021;cited November 05, 2021]. Available from: http://www.tousekiikai.or.jp/htm/03_info/doc03/20210820_antibody_cocktail_therapy.pdf

6. Nohara J, Nohara T, Abe N, et al. An experience of antibody cocktail therapy for a hemodialysis patient with COVID-19. Nihontouseki-ikaizasshi (in Japanese). [updated September 14, 2021; cited November 05, 2021]. Available from: ousekiikai.or.jp/htm/03_info/doc03/20210914_case_of_antibody_cocktail.pdf

7. Chung EYM, Palmer SC, Natale P, et al. Incidence and outcomes of COVID-19 in people with CKD: a systematic review and meta-analysis. Am J Kidney Dis. 2021 Aug 5. DOI: https://doi.org/10.1053/j.ajkd.2021.07.003.

8. Attias $P$, Sakhi H, Rieu P, et al. Antibody response to the BNT162b2 vaccine in maintenance hemodialysis patients. Kidney Int. 2021 Apr 19;99:1490-1492.

9. Glenn DA, Hedge A, Kotzen E, et al. Systematic review of safety and efficacy of COVID-19 vaccines in patients with kidney disease. Kidney Int Rep. 2021 May;6:1407-1410.

10. The Japanese Ministry of Health, Labour, and Welfare. Distribution of the combination of casirivimab and indevimab for medical facilities in Japan (Amendment). [updated October 1 , 2021;cited November 05, 2021]. Available from: https://www.mhlw.go.jp/content/000836895.pdf

\section{Figures}




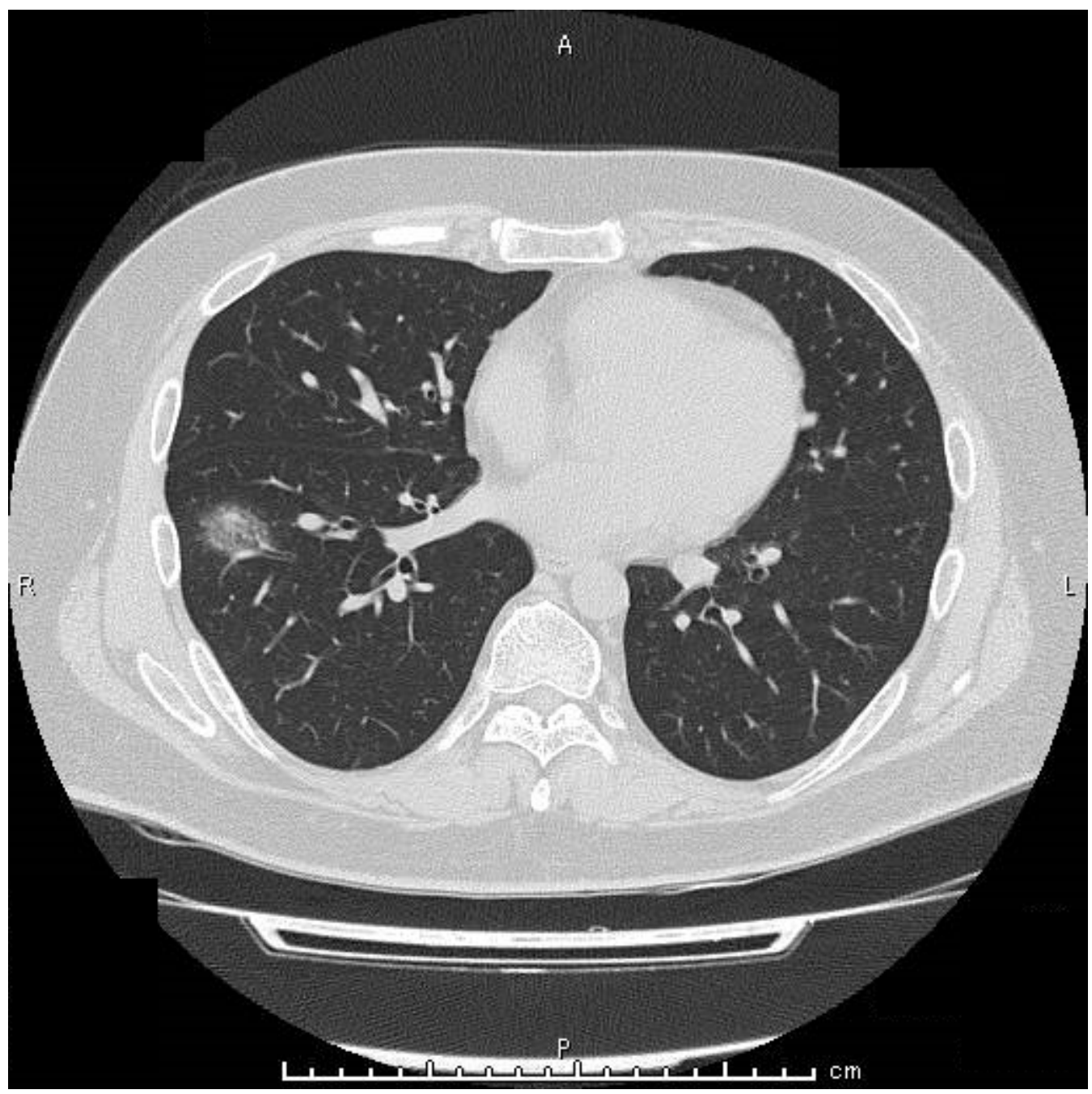

Figure 1

Plain computed tomography of the chest showed a focal ground-glass opacity in the right lower lobe. 